\title{
Does Rotator Cuff Repair Improve Psychologic Status and Quality of Life in Patients With Rotator Cuff Tear?
}

\author{
Chul-Hyun Cho MD, PhD, Kwang-Soon Song MD, \\ Ilseon Hwang MD, Jon J. P. Warner MD
}

Published online: 20 March 2015

(C) The Association of Bone and Joint Surgeons (B) 2015

\begin{abstract}
Background Recently, psychological status, patient-centered outcomes, and health-related quality of life (HRQoL) in patients with scheduled or who underwent orthopaedic surgeries have been emphasized. The relationship between preoperative psychological status and postoperative clinical outcome in patients with rotator cuff repair has not yet been investigated.
\end{abstract}

\section{Each author certifies that he or she, or a member of his or her} immediate family, has no commercial associations (eg, consultancies, stock ownership, equity interest, patent/licensing arrangements, etc) that might pose a conflict of interest in connection with the submitted article.

All ICMJE Conflict of Interest Forms for authors and Clinical Orthopaedics and Related Research ${ }^{\circledR}$ editors and board members are on file with the publication and can be viewed on request.

Each author certifies that his or her institution approved the human protocol for this investigation, that all investigations were conducted in conformity with ethical principles of research, and that informed consent for participation in the study was obtained.

\section{C.-H. Cho ( $₫)$}

Pain Research Center, Department of Orthopedic Surgery, Dongsan Medical Center, School of Medicine, Keimyung University, 56 Dalsung-ro, Jung-gu, Daegu 700-712, Korea e-mail: oscho5362@dsmc.or.kr

\section{K.-S. Song}

Department of Orthopedic Surgery, Dongsan Medical Center, School of Medicine, Keimyung University, Jung-gu, Daegu, Korea

I. Hwang

Department of Pathology, Dongsan Medical Center, School of Medicine, Keimyung University, Jung-gu, Daegu, Korea

\section{J. J. P. Warner}

Harvard Shoulder Service, Massachusetts General Hospital, Harvard Medical School, Boston, MA, USA
Questions/purposes The primary objective of this study was to investigate changes in psychological status (depression, anxiety, insomnia) and HRQoL after rotator cuff repair. The secondary objective was to assess whether preoperative depression, anxiety, and insomnia predict clinical outcome after rotator cuff repair.

Methods Forty-seven patients who underwent rotator cuff repair prospectively completed the visual analog scale (VAS) pain score, the UCLA Scale, the American Shoulder and Elbow Surgeons' Scale (ASES), the Hospital Anxiety and Depression Scale (HADS), the Pittsburgh Sleep Quality Index (PSQI), and the World Health Organization Quality-of-life Scale Abbreviated Version (WHOQOL-BREF) before surgery and at 3, 6, and 12 months after surgery. Repeated-measures analysis of variance was used to evaluate the serial changes in psychological parameters and outcome measurements. The chi-square test was also used to compare preoperative and postoperative prevalence of depression, anxiety, and insomnia. Finally, multiple regression analysis was applied to determine the relationship between preoperative psychological status and postoperative clinical outcome.

Results With surgery, depression, anxiety, and insomnia decreased, whereas quality of life increased. The mean HADS-D and HADS-A scores and the mean PSQI score decreased from $3.7 \pm 3.3,4.3 \pm 4.3$, and $6.6 \pm 3.6$, respectively, before surgery to $2.1 \pm 2.3,1.4 \pm 2.4$, and $4.2 \pm 3.3$, respectively, at 12 months after surgery (HADS-D mean difference 1.6 [95\% confidence interval $\{\mathrm{CI}\}, 0.6-2.6], \mathrm{p}=0.003$; HADS-A mean difference 2.9 [1.5-4.4], $\mathrm{p}<0.001$; PSQI mean difference 2.4 [1.3-3.4], $\mathrm{p}<0.001)$. The mean WHOQOL-BREF score increased from $60.4 \pm 11.0$ before surgery to $67.4 \pm 11.8$ at 12 months after surgery (mean difference $-7.0[95 \% \mathrm{CI}$, -10.7 to -3.4$], p<0.001)$. At 12 months after surgery, there were decreases in the prevalence of depression (six of 
47 [22.8\%] versus three of 47 [6.4\%], $\mathrm{p}=0.002)$, anxiety (11 of 47 [23.4\%] versus two of 47 [4.3\%], $\mathrm{p}=0.016$ ), and insomnia (33 of 47 [70.2\%] versus 20 of 47 [42.6\%], $\mathrm{p}=0.022$ ). Preoperative HADS-depression, HADS-anxiety, and PSQI scores did not correlate with the VAS pain score, UCLA, or ASES scores at 12 months after surgery. Conclusions Psychological status and HRQoL improved with decreasing pain and increasing functional ability from 3 months after surgery. Preoperative depression, anxiety, and insomnia did not predict poor outcome after rotator cuff repair. Our findings suggest that successful rotator cuff repair may improve psychological status and HRQoL. Level of Evidence Level II, prospective study.

\section{Introduction}

Rotator cuff tear is a common condition that causes pain and functional disability such as limited motion and weakness of the shoulder [10]. Although the currently preferred option is to provide nonoperative treatment to patients with symptomatic rotator cuff tears, patients whose condition does not resolve after nonoperative treatment are candidates for surgery [18]. Rotator cuff repair generally leads to a considerable decrease in pain and an increase in functional ability of the shoulder [3, 18]. However, the determinants of outcomes measured in patients with rotator cuff repair typically have been limited to aspects of physical health, especially surgeon-rated perceptions and measurements [15].

Several authors suggested that a complete understanding of clinical outcomes requires consideration of physical, psychological, and social factors [14, 24]. Perruccio et al. [20] noted that mental well-being is critical for understanding the relationship between physical health and selfrated health. Self-rated health, including psychological status, substantially predicts outcomes after total joint arthroplasty, above and beyond prior physical health [20]. Cho et al. [9] documented that psychological distress has a negative effect on outcome measurements in patients scheduled for rotator cuff repair. However, the impact of psychological predispositions, including depression, anxiety, and insomnia, on clinical outcomes after rotator cuff repair is still poorly understood. Also, it is not known whether rotator cuff repair improves psychological status.

Therefore, the primary objective of this study was to investigate changes in psychological status (depression, anxiety, insomnia) and health-related quality of life (HRQoL) after rotator cuff repair. We hypothesized that rotator cuff repair improves psychological status and HRQoL. The secondary objective was to assess whether preoperative depression, anxiety, and insomnia predict clinical outcome after rotator cuff repair.

\section{Patients and Methods}

\section{Study Subjects}

After obtaining approval from our institutional review board and written informed consent from all patients, we enrolled 47 patients undergoing rotator cuff repair at a tertiary care institution in our study between January 2010 and February 2011. Patients were included in the study if (1) their nonoperative treatment such as medication, injection, or physical therapy had failed after 3 months; (2) they were undergoing complete repair for a rotator cuff tear; (3) they had not undergone previous shoulder surgery; (4) they had no history of psychiatric disorders; and (5) they had followup examination until 12 months postoperatively. Candidates were excluded if (1) they were undergoing partial repair for irreparable rotator cuff tear; (2) they had undergone additional procedures such as biceps tenodesis, repair of a superior labrum anterior-toposterior tear, or distal clavicle resection; (3) they had undergone previous shoulder surgery; (4) they had a workers' compensation claim; or (5) they had any history of a psychiatric disorder. Of the 58 patients eligible for this study, 11 were excluded because all serial data were not obtained by loss of followup.

The mean age of the patients was $57 \pm 8$ years (range, 43-75 years), and there were 27 (57\%) women and 20 (43\%) men. Of these, $32(68 \%)$ underwent surgery on their dominant arm and $15(32 \%)$ underwent surgery on their nondominant arm. Seven (15\%) patients had a partial rotator cuff rear, seven (15\%) had a small tear, 17 (36\%) had a medium tear, seven (15\%) had a large tear, and nine (19\%) had a massive tear. The mean duration of symptoms was $25 \pm 36$ months (range, 3-120 months), and six patients $(13 \%)$ had preoperative stiffness. All procedures were performed by a single surgeon (C-HC); 37 (79\%) were arthroscopic and $10(21 \%)$ were miniopen rotator cuff repairs.

\section{Study Measures}

Study participants completed the visual analog scale (VAS) pain score, the UCLA scale [12], the American Shoulder and Elbow Surgeons' Scale (ASES) [22], the Hospital Anxiety and Depression Scale (HADS) [13], the Pittsburgh Sleep Quality Index (PSQI) [6], and the World Health Organization Quality of Life Scale Abbreviated Version (WHOQOL-BREF) [11] before surgery and at 3, 6, and 12 months after surgery. To minimize psychological distress associated with surgery, all questionnaires were completed at least 2 weeks before surgery. 
Hospital Anxiety and Depression Scale

The HADS consists of two seven-item subscales measuring depression (HADS-D) and anxiety (HADS-A) [19]. With high consistency and reliability, it has been commonly used as a screening tool for the detection of depression and anxiety in patients with musculoskeletal disorders [4, 13, 19]. Each item on the questionnaire is scored from 0 to 3 and this means that a patient can score between 0 and 21 for either anxiety or depression. Higher scores indicate greater likelihood of depression or anxiety. Recommended cutoffs are: 0 to $7=$ "normal"; 8 to $10=$ "mild case"; 11 to $14=$ "moderate case"; and 15 to $21=$ "severe case" $[8,19]$.

\section{Pittsburgh Sleep Quality Index}

The PSQI is a 19-item self-report instrument designed to measure sleep quality. It has been generally used for monitoring sleep disturbance in clinical practice $[2,6]$. This assessment measures seven categories: subjective sleep quality, sleep latency, sleep duration, habitual sleep efficiency, sleep disturbances, use of sleep medication, and daytime dysfunction [6]. Scores $>5$ indicate clinically meaningfully disturbed or poor sleep.

\section{World Health Organization Quality-of-life Scale Abbreviated Version}

The WHOQOL-BREF is an abbreviated generic quality-oflife scale developed through the World Health Organization. It contains 26 items and provides four domain scores (physical, psychological, social relationships, environment) that are each scored on a scale of 0 (poorest quality of life) to 100 (highest quality of life) [1, 11]. It has been established as a valid and reliable tool to assess quality of life in patients with musculoskeletal disorders $[1,11,17]$.

\section{Statistical Analysis}

The SPSS statistical package (Version 20.0; IBM, Armonk, NY, USA) was used for analysis and modeling of the data. The paired t-test and repeated-measures analysis of variance (ANOVA) were used to evaluate the changes in psychological parameters (HADS-D, HADS-A, PSQI scores) and outcome measurements (VAS pain score and UCLA, ASES, WHOQOL-BREF scores). Depression and anxiety based on the HADS-D and HADS-A were categorized as normal, mild, moderate, and severe using the described cutoff values. The chi-square test was used to compare preoperative and postoperative prevalence of depression, anxiety, and insomnia. Multiple linear regression analysis was applied to determine the association of preoperative HADS-D, HADS-A, and PSQI scores with 12month improvement in VAS pain score, UCLA, and ASES scores. The Pearson correlation, Kendall $\tau$ rank correlation, and Mann-Whitney U test were used to assess the correlation between outcome measurements and various parameters such as age, sex, involved side, duration of symptoms, tear size, preoperative stiffness, and repair technique. Two-tailed $\mathrm{p}$ values of $<0.05$ were considered significant.

On the basis of power analysis, the minimum sample size of patients-40 patients-was calculated using a $20 \%$ difference in ASES scores at an $\alpha$ level of 0.05 and $\beta$ level of 0.08 . However, 58 patients were included in the sample to ensure a sufficiently large sample to compensate for $30 \%$ possible dropout.

\section{Results}

With surgery, depression, anxiety, and insomnia decreased, whereas quality of life increased. The mean HADS-D and HADS-A scores and the mean PSQI score decreased from $3.7 \pm 3.3,4.3 \pm 4.3$, and $6.6 \pm 3.6$, respectively, before surgery to $2.1 \pm 2.3,1.4 \pm 2.4$, and $4.2 \pm 3.3$, respectively, at 12 months after surgery (HADS-D mean difference $1.6[95 \%$ confidence interval $\{\mathrm{CI}\}, 0.6-2.6]$, $\mathrm{p}=0.003$; HADS-A mean difference 2.9 [1.5-4.4], $\mathrm{p}<0.001 ; \quad$ PSQI mean difference 2.4 [1.3-3.4], $\mathrm{p}<0.001)$. The mean WHOQOL-BREF score increased from $60.4 \pm 11.0$ before surgery to $67.4 \pm 11.8$ at 12 months after surgery (mean difference -7.0 [95\% CI, -10.7 to -3.4$], \mathrm{p}<0.001$ ). Regarding serial change using repeated-measures ANOVA, we found improvement in HADS anxiety $(\mathrm{p}=0.023)$, PSQI $(\mathrm{p}=0.006)$, and WHOQOL-BREF $(p=0.036)$ scores as well as all outcome measurements, including VAS pain score $(\mathrm{p}<0.001)$, UCLA $(\mathrm{p}<0.001)$, and ASES scores $(\mathrm{p}<0.001)$ (Table 1). Although there was no overall serial change in HADS-depression score $(\mathrm{p}=0.148)$, there was a difference between preoperative HADS-depression score and the score at 3 months after surgery, demonstrated by the post hoc test $(\mathrm{p}=0.042)$ (Table 2). At 12 months after surgery, there were decreases in the prevalence of depression (six of 47 [22.8\%] versus three of 47 [6.4\%], $\mathrm{p}=0.002$ ), anxiety (11 of 47 [23.4\%] versus two of 47 [4.3\%], $\mathrm{p}=0.016$ ), and insomnia (33 of 47 [70.2\%] versus 20 of 47 [42.6\%], $\mathrm{p}=0.022$ ) (Table 3).

In multiple linear regression analysis, preoperative HADS-D, HADS-A, and PSQI scores did not correlate with the VAS pain score, UCLA, or ASES scores at 12 months 
Table 1. Serial changes in psychological and outcome measurements after rotator cuff repair

\begin{tabular}{|c|c|c|c|c|c|}
\hline \multirow[t]{2}{*}{ Measurement } & \multirow[t]{2}{*}{ Before surgery } & \multicolumn{3}{|l|}{ After surgery } & \multirow[t]{2}{*}{$\mathrm{p}$ value } \\
\hline & & At 3 months & At 6 months & At 12 months & \\
\hline VAS pain score & $6.7 \pm 1.6$ & $4.3 \pm 2.0$ & $2.9 \pm 2.1$ & $1.3 \pm 1.4$ & $<0.001 *$ \\
\hline UCLA score & $12.8 \pm 4.9$ & $24.3 \pm 4.7$ & $27.9 \pm 3.4$ & $31.0 \pm 3.3$ & $<0.001 *$ \\
\hline ASES score & $42.5 \pm 16.5$ & $60.3 \pm 16.3$ & $72.5 \pm 13.5$ & $87.2 \pm 10.9$ & $<0.001 *$ \\
\hline HADS-D score & $3.7 \pm 3.3$ & $2.4 \pm 3.2$ & $2.4 \pm 2.5$ & $2.1 \pm 2.3$ & 0.148 \\
\hline HADS-A score & $4.3 \pm 4.3$ & $2.5 \pm 4.2$ & $2.1 \pm 2.9$ & $1.4 \pm 2.4$ & $0.023 *$ \\
\hline PSQI score & $6.6 \pm 3.6$ & $5.6 \pm 3.9$ & $5.8 \pm 3.2$ & $4.2 \pm 3.3$ & $0.006^{*}$ \\
\hline WHOQOL-BREF score & $60.4 \pm 11.0$ & $62.4 \pm 12.3$ & $63.6 \pm 12.9$ & $67.4 \pm 11.8$ & $0.036 *$ \\
\hline
\end{tabular}

* Statistically significant; VAS = visual analog scale; ASES = American Shoulder and Elbow Surgeons' Scale; HADS-D = Hospital Anxiety and Depression Scale, depression subsection; HADS-A = Hospital Anxiety and Depression Scale, anxiety subsection; PSQI = Pittsburgh Sleep Quality Index; WHOQOL-BREF = World Health Organization Quality-of-life Scale Abbreviated Version.

Table 2. Interval analysis for change in depression using the post hoc test

\begin{tabular}{lll}
\hline HADS-D score & df & p value \\
\hline Preoperative to 3 months after surgery & 1 & $0.042^{*}$ \\
Preoperative to 6 months after surgery & 1 & 0.377 \\
Preoperative to 12 months after surgery & 1 & 0.574 \\
\hline
\end{tabular}

* Statistically significant; HADS-D = Hospital Anxiety and Depression Scale, depression subsection; $\mathrm{df}=$ degrees of freedom.

Table 3. Prevalence of depression, anxiety, and insomnia

\begin{tabular}{lccc}
\hline $\begin{array}{l}\text { Psychological } \\
\text { parameter }\end{array}$ & \multicolumn{2}{l}{ Number $(\%)$} & p value \\
\cline { 2 - 3 } & $\begin{array}{l}\text { Before } \\
\text { surgery }\end{array}$ & $\begin{array}{l}\text { 12 months } \\
\text { after surgery }\end{array}$ & \\
\hline $\begin{array}{l}\text { Depression } \\
\text { Normal }\end{array}$ & $41(87.2)$ & $44(93.6)$ & $0.002^{*}$ \\
Abnormal & $6(12.8)$ & $3(6.4)$ & \\
Mild & $4(8.5)$ & $3(6.4)$ & \\
Moderate & $2(4.3)$ & $0(0.0)$ & $0.016^{*}$ \\
Severe & $0(0.0)$ & $0(0.0)$ & \\
Anxiety & $36(76.6)$ & $45(95.7)$ & \\
Normal & $11(23.4)$ & $2(4.3)$ & \\
Abnormal & $7(14.9)$ & $2(4.3)$ & \\
Mild & $3(6.4)$ & $0(0.0)$ & \\
Moderate & $1(2.1)$ & $0(0.0)$ & \\
Severe & & & \\
Insomnia & $33(70.2)$ & $20(42.6)$ & \\
Yes & $14(29.8)$ & $27(57.4)$ & \\
No & &
\end{tabular}

* Statistically significant.

after surgery (Table 4). There was no correlation between outcome measurements at 12 months after surgery and various parameters such as age, sex, duration of symptoms, tear size, and repair technique, except that the involved side was correlated with VAS pain score $(\mathrm{p}=0.038)$ and preoperative stiffness had a positive correlation with UCLA and ASES scores $(p=0.041,0.041$; Table 5).

\section{Discussion}

The determinants of outcomes measured in patients with rotator cuff repair typically have been limited to aspects of physical health, especially surgeon-rated perceptions and measurements [15]. More recently, a greater emphasis has been placed on psychological status, patient-centered outcomes, and HRQoL [10, 15, 18, 20, 21]. We conducted a study to evaluate (1) changes in psychological status and HRQoL after rotator cuff repair; and (2) whether preoperative depression, anxiety, and insomnia predict clinical outcome after rotator cuff repair.

This study had a number of limitations. First, we did not have a control group and compare clinical outcomes, including psychological parameters and HRQoL, with those for a cohort undergoing nonoperative treatment. Second, the followup was relatively short and the dropout rate (19\%) was high. It might result in different outcomes, especially if this is the unhappy cohort that did not return because they were unsatisfied with surgery. Third, response bias cannot be excluded, because our assessment of depression, anxiety, and insomnia was based on participants' self-reports of their status obtained by a questionnaire. The minimal clinical importance difference (MCID) of the HADS and PSQI was not determined. However, the HADS and PSQI have been widely used as a screening instrument because of high internal consistency and test-retest reliability. Fourth, the power analysis had been based off the ASES score, not the psychological parameters because ours is a first study to determine psychological improvement after rotator cuff repair. Fifth, populations of our study 
Table 4. Effect of preoperative depression, anxiety, and insomnia on postoperative outcome measurements

\begin{tabular}{|c|c|c|c|}
\hline \multirow[t]{2}{*}{ Preoperative parameter } & \multicolumn{3}{|c|}{12 months after surgery } \\
\hline & VAS pain score & UCLA score & ASES score \\
\hline \multicolumn{4}{|l|}{ HADS-D score } \\
\hline Coefficient & -0.073 & -0.027 & 0.235 \\
\hline $95 \% \mathrm{CI}$ & -0.298 to 0.152 & -0.565 to 0.511 & -1.492 to 1.963 \\
\hline $\mathrm{p}$ value & 0.515 & 0.920 & 0.785 \\
\hline \multicolumn{4}{|l|}{ HADS-A score } \\
\hline Coefficient & 0.115 & -0.089 & -0.624 \\
\hline $95 \% \mathrm{CI}$ & -0.053 to 0.283 & -0.491 to 0.313 & -1.913 to 0.665 \\
\hline $\mathrm{p}$ value & 0.174 & 0.657 & 0.335 \\
\hline \multicolumn{4}{|l|}{ PSQI score } \\
\hline Coefficient & 0.040 & 0.001 & 0.386 \\
\hline $95 \% \mathrm{CI}$ & -0.082 to 0.163 & -0.295 to 0.293 & -1.330 to 0.558 \\
\hline $\mathrm{p}$ value & 0.664 & 0.992 & 0.415 \\
\hline
\end{tabular}

VAS = visual analog scale; ASES = American Shoulder and Elbow Surgeons' Scale; HADS-D = Hospital Anxiety and Depression Scale, depression subsection; $\mathrm{CI}=$ confidence interval; HADS-A = Hospital Anxiety and Depression Scale, anxiety subsection; PSQI = Pittsburgh Sleep Quality Index; UCLA = University of California Los Angeles Scale.

Table 5. Correlation between variables and postoperative outcome measurements

\begin{tabular}{llllrrrrr}
\hline Variables & & $\begin{array}{l}\text { VAS pain } \\
\text { score }\end{array}$ & $\begin{array}{l}\text { UCLA } \\
\text { score }\end{array}$ & $\begin{array}{l}\text { ASES } \\
\text { score }\end{array}$ & \multicolumn{1}{l}{$\begin{array}{l}\text { HADS-D } \\
\text { score }\end{array}$} & $\begin{array}{l}\text { HADS-A } \\
\text { score }\end{array}$ & $\begin{array}{l}\text { PSQI } \\
\text { score }\end{array}$ & $\begin{array}{l}\text { WHOQOL-BREF } \\
\text { score }\end{array}$ \\
\hline Age & Pearson correlation & 0.011 & 0.155 & -0.006 & -0.053 & 0.055 & 0.264 & -0.107 \\
& p value & 0.942 & 0.298 & 0.969 & 0.725 & 0.716 & 0.073 & 0.476 \\
Sex & p value & 0.677 & 0.142 & 0.170 & 0.054 & 0.194 & 0.255 & 0.111 \\
Involved side & p value & $0.038^{*}$ & 0.057 & 0.212 & 0.339 & 0.979 & 0.512 & 0.810 \\
Duration of symptoms & Pearson correlation & 0.093 & 0.064 & 0.026 & -0.189 & -0.194 & -0.122 & 0.081 \\
Tear size & p value & 0.536 & 0.668 & 0.860 & 0.203 & 0.191 & 0.415 & 0.590 \\
Preoperative stiffness & Pearson correlation & 0.267 & -0.177 & -0.256 & -0.023 & -0.115 & -0.019 & -0.048 \\
Repair technique & p value & 0.070 & 0.233 & 0.082 & 0.876 & 0.441 & 0.902 & 0.750 \\
\hline
\end{tabular}

* Statistically significant; VAS = visual analog scale; ASES = American Shoulder and Elbow Surgeons' Scale; HADS-D = Hospital Anxiety and Depression Scale, depression subsection; HADS-A = Hospital Anxiety and Depression Scale, anxiety subsection; PSQI = Pittsburgh Sleep Quality Index; WHOQOL-BREF = World Health Organization Quality-of-life Scale Abbreviated Version.

were quite heterogeneous. We included patients with small subgroups ranging from partial to massive rotator cuff tears and patients with either arthroscopic or miniopen rotator cuff repair. Sixth, we did not assess the structural integrity of repaired rotator cuffs at followup evaluations or the correlation between structural integrity of repaired tendon and clinical outcome measurements, including psychological parameters and HRQoL.

In terms of strength, it is meaningful that our study is the first, to our knowledge, longitudinal prospective cohort study to report changes in psychological status such as depression, anxiety, insomnia, and HRQoL after rotator cuff repair and to investigate the relationship between preoperative psychological status and postoperative clinical outcome.
Our findings support our hypothesis that rotator cuff repair improves psychological status (depression, anxiety, insomnia) and HRQoL. Our findings also revealed that it is not only a matter of improved pain and physical function, but also of improvement of mental health and general health in these patients. In addition, the degree of both depression and anxiety markedly improved by 12 months after surgery. By that point, only three patients (6.4\%) had mild depression and only two patients $(4.3 \%)$ had mild anxiety. Although insomnia prevalence of $42.6 \%$ at 12 months after surgery seems to be high compared with $25.0 \%$ in a normal population reported by Cho et al. [8], this decreased by 12 months after surgery. These findings imply that preoperative depression, anxiety, and insomnia are reversible with appropriate treatment. 
More recently, patients' expectations and preoperative psychological predisposition have been discussed as determinants of postoperative pain, function, and HRQOL [1, $20,23]$. Several researchers have shown that intense preoperative psychological distress may also produce dissatisfaction for treatment and disturb postoperative recovery in major orthopaedic procedures such as total joint replacement or spinal surgery $[1,5,20,23]$. They suggested that preoperative anxiety and depression are independent predictors of postoperative clinical outcome. In contrast, multivariate analysis of our data showed that preoperative HADS-D, HADS-A, and PSQI scores did not correlate with VAS pain score, UCLA, or ASES scores at 12 months after surgery. This finding means that preoperative depression, anxiety, and insomnia did not predict poor outcome after rotator cuff repair.

Previous studies have shown that various preoperative factors, including age, sex, duration of symptoms, preoperative stiffness, tear size, fatty infiltration, and repair technique, predict clinical outcomes after rotator cuff repair $[3,7,10,16]$. In our study, there was no significant correlation between postoperative clinical outcome and various parameters such as age, sex, duration of symptoms, tear size, and repair technique, except involved side and preoperative stiffness. Although involvement of the dominant arm had higher VAS pain score, we believe that this finding may not be that meaningful, because there was no correlation with other outcome measurements such as UCLA, ASES, and WHOQOL-BREF scores. Preoperative stiffness was positively correlated with UCLA and ASES scores. However, interpretation of this finding was difficult because the followup was short. For a better interpretation of the correlation, a study of long-term followup is needed.

In conclusion, this study demonstrated that patients had improvement in depression, anxiety, sleep disturbance, health status, pain, and functional status after rotator cuff repair. Interestingly, preoperative depression, anxiety, and insomnia did not appear to affect 1-year outcome measures. Future long-term randomized controlled trials should be considered for further evaluation of change in psychological status and quality of life in patients with rotator cuff tear treated conservatively versus surgically.

Acknowledgments We thank Eun-Ji Jeon, Do-Yeon Kim, and MinJee Kim for their support with data collection.

\section{References}

1. Ackerman IN, Graves SE, Bennell KL, Osborne RH. Evaluating quality of life in hip and knee replacement: psychometric properties of the World Health Organization Quality of Life short version instrument. Arthritis Rheum. 2006;55:583-590.
2. Backhaus J, Junghanns K, Broocks A, Riemann D, Hohagen F. Test-retest reliability and validity of the Pittsburgh Sleep Quality Index in primary insomnia. J Psychosom Res. 2002;53:737-740.

3. Baysal D, Balyk R, Otto D, Luciak-Corea C, Beaupre L. Functional outcome and health-related quality of life after surgical repair of full-thickness rotator cuff tear using a mini-open technique. Am J Sports Med. 2005;33:1346-1355.

4. Bjelland I, Dahl AA, Haug TT, Neckelmann D. The validity of the Hospital Anxiety and Depression Scale. An updated literature review. J Psychosom Res. 2002;52:69-77.

5. Blackburn J, Qureshi A, Amirfeyz R, Bannister G. Does preoperative anxiety and depression predict satisfaction after total knee replacement? Knee. 2012;19:522-524.

6. Buysse DJ, Reynolds CF 3rd, Monk TH, Berman SR, Kupfer DJ. The Pittsburgh Sleep Quality Index: a new instrument for psychiatric practice and research. Psychiatry Res. 1989;28:193-213.

7. Charousset C, Grimberg J, Duranthon LD, Bellaiche L, Petrover D, Kalra K. The time for functional recovery after arthroscopic rotator cuff repair: correlation with tendon healing controlled by computed tomography arthrography. Arthroscopy. 2008;24: 25-33.

8. Cho CH, Jung SW, Park JY, Song KS, Yu KI. Is shoulder pain for three months or longer correlated with depression, anxiety, and sleep disturbance? J Shoulder Elbow Surg. 2013;22:222-228.

9. Cho CH, Seo HJ, Bae KC, Lee KJ, Hwang I, Warner JJ. The impact of depression and anxiety on self-assessed pain, disability, and quality of life in patients scheduled for rotator cuff repair. $J$ Shoulder Elbow Surg. 2013;22:1160-1166.

10. Chung SW, Park JS, Kim SH, Shin SH, Oh JH. Quality of life after arthroscopic rotator cuff repair: evaluation using SF-36 and an analysis of affecting clinical factors. Am J Sports Med. 2012;40:631-639.

11. Development of the World Health Organization WHOQOLBREF quality of life assessment. The WHOQOL Group. Psychol Med. 1998;28:551-558.

12. Ellman H, Hanker G, Bayer M. Repair of the rotator cuff. Endresult study of factors influencing reconstruction. $J$ Bone Joint Surg Am. 1986;68:1136-1144.

13. Härter M, Reuter K, Gross-Hardt K, Bengel J. Screening for anxiety, depressive and somatoform disorders in rehabilitationvalidity of HADS and GHQ-12 in patients with musculoskeletal disease. Disabil Rehabil. 2001;23:737-744.

14. Hoffman BM, Papas RK, Chatkoff DK, Kerns RD. Meta-analysis of psychological interventions for chronic low back pain. Health Psychol. 2007;26:1-9.

15. Hultenheim Klintberg I, Karlsson J, Svantesson U. Health-related quality of life, patient satisfaction, and physical activity 8-11 years after arthroscopic subacromial decompression. $J$ Shoulder Elbow Surg. 2011;20:598-608.

16. McRae S, Leiter J, Walmsley C, Rehsia S, Macdonald P. Relationship between self-reported shoulder function/quality of life, body mass index, and other contributing factors in patients awaiting rotator cuff repair surgery. J Shoulder Elbow Surg. 2011;20:57-61.

17. Min SK, Kim KI, Lee CI, Jung YC, Suh SY, Kim DK. Development of the Korean versions of WHO Quality of Life scale and WHOQOL-BREF. Qual Life Res. 2002;11:593-600.

18. Mohtadi NG, Hollinshead RM, Sasyniuk TM, Fletcher JA, Chan DS, Li FX. A randomized clinical trial comparing open to arthroscopic acromioplasty with mini-open rotator cuff repair for full-thickness rotator cuff tears: disease-specific quality of life outcome at an average 2-year follow-up. Am J Sports Med. 2008;36:1043-1051.

19. Pallant JF, Bailey CM. Assessment of the structure of the Hospital Anxiety and Depression Scale in musculoskeletal patients. Health Qual Life Outcomes. 2005;3:82. 
20. Perruccio AV, Davis AM, Hogg-Johnson S, Badley EM. Importance of self-rated health and mental well-being in predicting health outcomes following total joint replacement surgery for osteoarthritis. Arthritis Care Res (Hoboken). 2011;63:973-981.

21. Piitulainen K, Ylinen J, Kautiainen H, Häkkinen A. The relationship between functional disability and health-related quality of life in patients with a rotator cuff tear. Disabil Rehabil. 2012;34:2071-2075.

22. Richards RR, An KN, Bigliani LU, Friedman RJ, Gartsman GM, Gristina AG, Iannotti JP, Mow VC, Sidles JA, Zuckerman JD. A standardized method for the assessment of shoulder function. $J$ Shoulder Elbow Surg. 1994;3:347-352.

23. Sinikallio S, Aalto T, Airaksinen O, Lehto SM, Kröger H, Viinamäki $H$. Depression is associated with a poorer outcome of lumbar spinal stenosis surgery: a two-year prospective follow-up study. Spine (Phila Pa 1976). 2011;36:677-682.

24. Vranceanu AM, Barsky A, Ring D. Psychosocial aspects of disabling musculoskeletal pain. J Bone Joint Surg Am. 2009;91: 2014-2018. 